\title{
Stability and Hopf Bifurcation in a Delayed SEIRS Worm Model in Computer Network
}

\author{
Zizhen Zhang ${ }^{1,2}$ and Huizhong Yang ${ }^{1}$ \\ ${ }^{1}$ Key Laboratory of Advanced Process Control for Light Industry (Ministry of Education), Jiangnan University, Wuxi 214122, China \\ ${ }^{2}$ School of Management Science and Engineering, Anhui University of Finance and Economics, Bengbu 233030, China \\ Correspondence should be addressed to Huizhong Yang; yhz@jiangnan.edu.cn
}

Received 8 June 2013; Revised 4 October 2013; Accepted 7 October 2013

Academic Editor: Piermarco Cannarsa

Copyright (c) 2013 Z. Zhang and H. Yang. This is an open access article distributed under the Creative Commons Attribution License, which permits unrestricted use, distribution, and reproduction in any medium, provided the original work is properly cited.

A delayed SEIRS epidemic model with vertical transmission in computer network is considered. Sufficient conditions for local stability of the positive equilibrium and existence of local Hopf bifurcation are obtained by analyzing distribution of the roots of the associated characteristic equation. Furthermore, the direction of the local Hopf bifurcation and the stability of the bifurcating periodic solutions are determined by using the normal form theory and center manifold theorem. Finally, a numerical example is presented to verify the theoretical analysis.

\section{Introduction}

Since the pioneering work of Kephart and White $[1,2]$, classical epidemiological models have been used to understand and predict virus propagations in computer network by many authors [3-10]. In [3], Thommes and Coates proposed a modified version of the SEI model to predict the virus propagation in a network. In [5], Yuan and Chen proposed an e-SEIR model and studied the behavior of virus propagation with the presence of antivirus countermeasures. Yuan and Chen [5] supposed that the recovered computers have a permanent immunization period and can no longer be infected. Considering that there is no permanent recovery from the virus till a node is attached to the computer network, Mishra and Pandey [6] proposed the following epidemic model for the transmission of worms with vertical transmission:

$$
\begin{gathered}
\frac{d S(t)}{d t}=b-\beta I(t) S(t)-p b E(t)-q b I(t)-d S(t)+\zeta R(t), \\
\frac{d E(t)}{d t}=\beta I(t) S(t)+p b E(t)+q b I(t)-\varepsilon E(t)-d E(t),
\end{gathered}
$$

$$
\begin{gathered}
\frac{d I(t)}{d t}=\varepsilon E(t)-\gamma I(t)-d I(t)-\eta I(t), \\
\frac{d R(t)}{d t}=\gamma I(t)-d R(t)-\zeta R(t),
\end{gathered}
$$

where $S(t), E(t), I(t)$, and $R(t)$ denote the numbers of nodes at time $t$ in susceptible, exposed, infectious and recovered states, respectively. $b$ is the recruitment rate of susceptible nodes to the computer network. $d$ is the crashing rate of nodes due to the reason other than the attack of worms. $p$ and $q$ are the fractions of the new nodes from the exposed and the infectious classes, respectively, that are introduced into the exposed class. $\beta$ is the transmission rate. $\varepsilon, \gamma$, and $\zeta$ are the state transition rates. $\eta$ is the crashing rate of nodes due to the attack of worms. Mishra and Pandey [6] discussed the characteristic of the basic reproduction number and investigated the global stability of system (1) by constructing a new Liyapunov function.

It is well known that time delays can play a complicated role on the dynamics of a system. They can cause the stable equilibrium of a system to become unstable and make a system bifurcate periodic solutions. Dynamical systems 
with delay have been studied by many scholars [11-20]. Motivated by the works above and considering that the antivirus software may use a period to clean the worms in one node and that the recovered nodes may have a temporary immunity period due to the antivirus software, we consider the following delayed system in this paper:

$$
\begin{aligned}
\frac{d S(t)}{d t}= & b-\beta I(t) S(t)-p b E(t)-q b I(t)-d S(t) \\
& +\zeta R\left(t-\tau_{2}\right) \\
\frac{d E(t)}{d t}= & \beta I(t) S(t)+p b E(t)+q b I(t)-\varepsilon E(t) \\
& -d E(t) \\
\frac{d I(t)}{d t}= & \varepsilon E(t)-\gamma I\left(t-\tau_{1}\right)-d I(t)-\eta I(t), \\
\frac{d R(t)}{d t} & =\gamma I\left(t-\tau_{1}\right)-d R(t)-\zeta R\left(t-\tau_{2}\right)
\end{aligned}
$$

where $\tau_{1}$ is the period to clean the worms in one node and $\tau_{2}$ is the temporary immunity period. For the convenience of analysis, throughout this paper, we assume that the period to clean the worms in one node and the temporary immunity period are the same.

Let $\tau_{1}=\tau_{2}=\tau$, and then system (2) becomes the following form:

$$
\begin{aligned}
\frac{d S(t)}{d t}= & b-\beta I(t) S(t)-p b E(t)-q b I(t)-d S(t) \\
& +\zeta R(t-\tau) \\
\frac{d E(t)}{d t}= & \beta I(t) S(t)+p b E(t)+q b I(t)-\varepsilon E(t) \\
& -d E(t), \\
\frac{d I(t)}{d t}= & \varepsilon E(t)-\gamma I(t-\tau)-d I(t)-\eta I(t) \\
\frac{d R(t)}{d t} & =\gamma I(t-\tau)-d R(t)-\zeta R(t-\tau) .
\end{aligned}
$$

The main purpose of this paper is to investigate the effects of the time delay on the dynamics of system (3). We study the stability of the positive equilibrium of system (3) and find the critical value of the time delay where a Hopf bifurcation occurs. We also study the properties of the Hopf bifurcation such as direction and stability.

This paper is organized as follows. In Section 2, we investigate local stability of the positive equilibrium and obtain the sufficient conditions for the existence of local Hopf bifurcation. In Section 3, we determine the direction and the stability of the Hopf bifurcation by using the normal form theory and center manifold theorem. In order to testify the theoretical analysis, a numerical example is presented in Section 4.

\section{Stability and Existence of Local Hopf Bifurcation}

It is not difficult to verify that if

$$
\begin{gathered}
R_{0}=\frac{b \beta \varepsilon+b d q \varepsilon+b d p(d+\gamma+\eta)}{d(d+\varepsilon)(d+\gamma+\eta)}>1, \\
(d+\varepsilon)(d+\gamma+\eta)>p b(d+\gamma+\eta)+q b \varepsilon,
\end{gathered}
$$

system (2) has a unique positive equilibrium $D_{*}\left(S_{*}, E_{*}\right.$, $\left.I_{*}, R_{*}\right)$, where

$$
\begin{gathered}
S_{*}=\frac{(d+\varepsilon)(d+\gamma+\eta)-p b(d+\gamma+\eta)-q b \varepsilon}{\beta \varepsilon}, \\
I_{*}=(d+\zeta)[b \beta \varepsilon+d(p b(d+\gamma+\eta) \\
\quad+q b \varepsilon-(d+\varepsilon)(d+\gamma+\eta))] \\
\quad \times(\beta(d+\varepsilon)(d+\zeta)(d+\gamma+\eta)-\beta \gamma \varepsilon \zeta)^{-1}, \\
E_{*}=\frac{d+\gamma+\eta}{\varepsilon} I_{*}, \quad R_{*}=\frac{\gamma}{d+\zeta} I_{*} .
\end{gathered}
$$

$R_{0}$ is called the basic reproduction number.

The Jacobian matrix of system (3) about the positive equilibrium $D_{*}$ is

$$
J\left(D_{*}\right)
$$

$$
=\left(\begin{array}{cccc}
\lambda-a_{11} & -a_{12} & -a_{12} & -b_{14} e^{-\lambda \tau} \\
-a_{21} & \lambda-a_{22} & -a_{23} & 0 \\
0 & -a_{32} & \lambda-a_{33}-b_{33} e^{-\lambda \tau} & 0 \\
0 & 0 & -b_{43} e^{-\lambda \tau} & \lambda-a_{44}-b_{43} e^{-\lambda \tau}
\end{array}\right),
$$

where

$$
\begin{gathered}
a_{11}=-\left(d+\beta I_{*}\right), \quad a_{12}=-p b, \\
a_{13}=-\left(q b+\beta S_{*}\right), \quad a_{21}=\beta I_{*}, \\
a_{22}=p b-d-\varepsilon, \quad a_{23}=q b+\beta S_{*}, \\
a_{32}=\varepsilon, \quad a_{33}=-(d+\eta), \\
a_{44}=-d, \quad b_{14}=\zeta, \quad b_{33}=-\gamma, \\
b_{43}=\gamma, \quad b_{44}=-\zeta .
\end{gathered}
$$

Thus, the characteristic equation of system (2) at the positive equilibrium $D_{*}$ is

$$
\begin{aligned}
\lambda^{4} & +A_{3} \lambda^{3}+A_{2} \lambda^{2}+A_{1} \lambda+A_{0} \\
& +\left(B_{3} \lambda^{3}+B_{2} \lambda^{2}+B_{1} \lambda+B_{0}\right) e^{-\lambda \tau} \\
& +\left(C_{2} \lambda^{2}+C_{1} \lambda+C_{0}\right) e^{-2 \lambda \tau}=0
\end{aligned}
$$

where

$$
\begin{aligned}
A_{0}= & a_{11} a_{44}\left(a_{22} a_{33}-a_{23} a_{32}\right)+a_{21} a_{44}\left(a_{13} a_{32}-a_{12} a_{33}\right), \\
A_{1}= & \left(a_{33}+a_{44}\right)\left(a_{12} a_{21}-a_{11} a_{22}\right)+a_{23} a_{32}\left(a_{11}+a_{44}\right) \\
& -a_{33} a_{44}\left(a_{11}+a_{22}\right)+a_{13} a_{21} a_{32},
\end{aligned}
$$




$$
\begin{gathered}
A_{2}=\left(a_{11}+a_{22}\right)\left(a_{33}+a_{44}\right)+a_{11} a_{22}+a_{33} a_{44}-a_{12} a_{21} \\
-a_{23} a_{32}, \\
A_{3}=-\left(a_{11}+a_{22}+a_{33}+a_{44}\right), \\
B_{0}=\left(a_{11} a_{22}-a_{12} a_{21}\right)\left(a_{33} b_{44}+a_{44} b_{33}\right) \\
\quad+\left(a_{13} a_{21}-a_{11} a_{23}\right) a_{32} b_{44}, \\
B_{1}=\left(b_{33}+b_{44}\right)\left(a_{12} a_{21}-a_{11} a_{22}\right)+a_{23} a_{32} b_{44} \\
-\left(a_{11}+a_{22}\right)\left(a_{33} b_{44}+a_{44} b_{33}\right), \\
B_{2}=\left(a_{11}+a_{22}\right)\left(b_{33}+b_{44}\right)+a_{33} b_{44}+a_{44} b_{33}, \\
B_{3}=-\left(b_{33}+b_{44}\right), \quad C_{2}=b_{33} b_{44}, \\
\quad C_{1}=b_{33} b_{44}\left(a_{11}+a_{22}\right), \\
C_{0}=\left(a_{11} a_{22}-a_{12} a_{21}\right) b_{33} b_{44}-a_{21} a_{32} b_{14} b_{43} .
\end{gathered}
$$

Multiplying $e^{\lambda \tau}$ on both sides of (8), it is easy to get

$$
\begin{aligned}
B_{3} \lambda^{3} & +B_{2} \lambda^{2}+B_{1} \lambda+B_{0} \\
& +\left(\lambda^{4}+A_{3} \lambda^{3}+A_{2} \lambda^{2}+A_{1} \lambda+A_{0}\right) e^{\lambda \tau} \\
& +\left(C_{2} \lambda^{2}+C_{1} \lambda+C_{0}\right) e^{-\lambda \tau}=0 .
\end{aligned}
$$

When $\tau=0$, (10) reduces to

$$
\lambda^{4}+A_{13} \lambda^{3}+A_{12} \lambda^{2}+A_{11} \lambda+A_{10}=0,
$$

where

$$
\begin{array}{cc}
A_{10}=A_{0}+B_{0}+C_{0}, & A_{11}=A_{1}+B_{1}+C_{1}, \\
A_{12}=A_{2}+B_{2}+C_{2}, & A_{13}=A_{3}+B_{3} .
\end{array}
$$

By the Routh-Hurwitz criterion, the sufficient conditions for all roots of (11) to have a negative real part given in the following form:

$$
\begin{gathered}
D_{1}=A_{13}>0, \\
D_{2}=\operatorname{det}\left(\begin{array}{cc}
A_{13} & 1 \\
A_{11} & A_{12}
\end{array}\right)>0, \\
D_{3}=\operatorname{det}\left(\begin{array}{ccc}
A_{13} & 1 & 0 \\
A_{11} & A_{12} & A_{13} \\
0 & A_{10} & A_{11}
\end{array}\right)>0, \\
D_{4}=\operatorname{det}\left(\begin{array}{cccc}
A_{13} & 1 & 0 & 0 \\
A_{11} & A_{12} & A_{13} & 1 \\
0 & A_{10} & A_{11} & A_{12} \\
0 & 0 & 0 & A_{10}
\end{array}\right)>0 .
\end{gathered}
$$

Thus, if the condition $\left(H_{1}\right)$ holds, which means that (13) and (14) are satisfied, the positive equilibrium $D_{*}$ is locally asymptotically stable in the absence of delay.

For $\tau>0$, let $\lambda=i \omega(\omega>0)$ be a root of (10). Then, we can get

$$
\begin{aligned}
& \left(\omega^{4}-\left(A_{2}+C_{2}\right) \omega^{2}+A_{0}+C_{0}\right) \cos \tau \omega \\
& \quad-\left(\left(A_{1}-C_{1}\right) \omega-A_{3} \omega^{3}\right) \sin \tau \omega=B_{2} \omega^{2}-B_{0}, \\
& \left(\omega^{4}-\left(A_{2}-C_{2}\right) \omega^{2}+A_{0}-C_{0}\right) \sin \tau \omega \\
& \quad+\left(\left(A_{1}+C_{1}\right) \omega-A_{3} \omega^{3}\right) \cos \tau \omega=B_{3} \omega^{3}-B_{1} \omega .
\end{aligned}
$$

Then, we can get

$$
\begin{aligned}
& \sin \tau \omega=\frac{p_{7} \omega^{7}+p_{5} \omega^{5}+p_{3} \omega^{3}+p_{1} \omega}{\omega^{8}+q_{6} \omega^{6}+q_{4} \omega^{4}+q_{2} \omega^{2}+q_{0}}, \\
& \cos \tau \omega=\frac{p_{6} \omega^{6}+p_{4} \omega^{4}+p_{2} \omega^{2}+p_{0}}{\omega^{8}+q_{6} \omega^{6}+q_{4} \omega^{4}+q_{2} \omega^{2}+q_{0}},
\end{aligned}
$$

where

$$
\begin{gathered}
p_{0}=B_{0}\left(C_{0}-A_{0}\right), \quad p_{1}=\left(A_{1}+C_{1}\right) B_{0}-\left(A_{0}+C_{0}\right) B_{1}, \\
p_{2}=\left(A_{2}-C_{2}\right) B_{0}+\left(A_{0}-C_{0}\right) B_{2}+\left(C_{1}-A_{1}\right) B_{1}, \\
p_{3}=\left(A_{0}+C_{0}\right) B_{3}-\left(A_{1}+C_{1}\right) B_{2}+\left(A_{2}+C_{2}\right) B_{1}-A_{3} B_{0}, \\
p_{4}=\left(A_{1}-C_{1}\right) B_{3}-\left(A_{2}-C_{2}\right) B_{2}+A_{3} B_{1}-B_{0}, \\
p_{5}=A_{3} B_{2}-B_{1}-\left(A_{2}+C_{2}\right) B_{3}, \\
p_{6}=B_{2}-A_{3} B_{3}, \quad p_{7}=B_{3}, \\
q_{0}=A_{0}^{2}-C_{0}^{2}, \quad q_{2}=A_{1}^{2}-C_{1}^{2}-2 A_{0} A_{2}+2 C_{0} C_{2}, \\
q_{4}=A_{2}^{2}-C_{2}^{2}+2 A_{0}-2 A_{1} A_{3}, \quad q_{6}=A_{3}^{2}-2 A_{2} .
\end{gathered}
$$

It is well known that $\sin ^{2} \tau \omega+\cos ^{2} \tau \omega=1$. Thus, we have

$$
\begin{aligned}
& \omega^{16}+e_{7} \omega^{14}+e_{6} \omega^{12}+e_{5} \omega^{10}+e_{4} \omega^{8}+e_{3} \omega^{6}+e_{2} \omega^{4} \\
& +e_{1} \omega^{2}+e_{0}=0,
\end{aligned}
$$

where

$$
\begin{gathered}
e_{0}=q_{0}^{2}-p_{0}^{2}, \quad e_{1}=2 q_{0} q_{2}-2 p_{0} p_{2}-p_{1}^{2}, \\
e_{2}=q_{2}^{2}+2 q_{0} q_{4}-p_{2}^{2}-2 p_{0} p_{4}-2 p_{1} p_{3}, \\
e_{3}=2 q_{0} q_{6}+2 q_{2} q_{4}-p_{3}^{2}-2 p_{0} p_{6}-2 p_{1} p_{5}-2 p_{2} p_{4}, \\
e_{4}=q_{4}^{2}+2 q_{0}+2 q_{2} q_{6}-p_{4}^{2}-2 p_{1} p_{7}-2 p_{2} p_{6}-2 p_{3} p_{5}, \\
e_{5}=2 q_{2}+2 q_{4} q_{6}-2 p_{3} p_{7}-2 p_{4} p_{6}-p_{5}^{2}, \\
e_{6}=q_{6}^{2}-p_{6}^{2}+2 q_{4}-2 p_{5} p_{7}, \quad e_{7}=2 q_{6}-p_{7}^{2} .
\end{gathered}
$$

Let $\omega^{2}=v$, and then (18) becomes

$$
\begin{aligned}
v^{8} & +e_{7} v^{7}+e_{6} v^{6}+e_{5} v^{5}+e_{4} v^{4}+e_{3} v^{3}+e_{2} v^{2} \\
& +e_{1} v+e_{0}=0 .
\end{aligned}
$$


In order to give the main results in this paper, we made the following assumption.

$\left(\mathrm{H}_{2}\right)$ Equation (20) has at least one positive real root.

Suppose that the condition $\left(\mathrm{H}_{2}\right)$ holds. Without loss of generality, we suppose that (20) has eight positive real roots, which are denoted as $v_{1}, v_{2}, \ldots, v_{8}$, respectively. Then, (18) has eight positive roots $\omega_{k}=\sqrt{v_{k}}, k=1,2, \ldots, 8$. For every fixed $\omega_{k}$, the corresponding critical value of time delay is

$$
\begin{array}{r}
\tau_{k}^{(j)}=\frac{1}{\omega_{k}} \operatorname{arc} \cos \frac{p_{6} \omega^{6}+p_{4} \omega_{k}^{4}+p_{2} \omega_{k}^{2}+p_{0}}{\omega^{8}+q_{6} \omega_{k}^{6}+q_{4} \omega_{k}^{4}+q_{2} \omega_{k}^{2}+q_{0}}+\frac{2 j \pi}{\omega_{k}}, \\
k=1,2, \ldots, 8, j=0,1,2, \ldots
\end{array}
$$

Let

$$
\begin{gathered}
\tau_{0}=\min \left\{\tau_{k}^{(0)}\right\}, \quad k \in\{1,2, \ldots, 8\}, \\
\omega_{0}=\left.\omega_{k}\right|_{\tau=\tau_{0}} .
\end{gathered}
$$

Taking the derivative of with respect to (10), it is easy to obtain

$$
\begin{aligned}
{\left[\frac{d \lambda}{d \tau}\right]^{-1}=} & \left(3 B_{3} \lambda^{2}+2 B_{2} \lambda+B_{1}+\left(2 C_{2} \lambda+C_{1}\right) e^{-\lambda \tau}\right. \\
& \left.+\left(4 \lambda^{3}+3 A_{3} \lambda^{2}+2 A_{2} \lambda+A_{1}\right) e^{\lambda \tau}\right) \\
& \times\left(\left(C_{2} \lambda^{3}+C_{1} \lambda^{2}+C_{0} \lambda\right) e^{-\lambda \tau}\right. \\
& \left.-\left(\lambda^{5}+A_{3} \lambda^{4}+A_{2} \lambda^{3}+A_{1} \lambda^{2}+A_{0} \lambda\right) e^{\lambda \tau}\right)^{-1} \\
& -\frac{\tau}{\lambda}
\end{aligned}
$$

Then, we have

$$
\begin{gathered}
\operatorname{Re}\left[\frac{d \lambda}{d \tau}\right]_{\tau=\tau_{0}}^{-1}=\frac{P_{R} Q_{R}+P_{I} Q_{I}}{Q_{R}^{2}+Q_{I}^{2}}, \\
P_{R}=\left(A_{1}+C_{1}-3 A_{3} \omega_{0}^{2}\right) \cos \tau_{0} \omega_{0} \\
+2\left(2 \omega_{0}^{3}-\left(A_{2}-C_{2}\right) \omega_{0}\right) \sin \tau_{0} \omega_{0}-3 B_{3} \omega_{0}^{2}+B_{1}, \\
P_{I}=\left(A_{1}-C_{1}-3 A_{3} \omega_{0}^{2}\right) \sin \tau_{0} \omega_{0} \\
+2\left(\omega_{0}^{3}-\left(A_{2}+C_{2}\right) \omega_{0}\right) \cos \tau_{0} \omega_{0}+2 B_{2} \omega_{0}, \\
Q_{R}=\left(A_{1} \omega_{0}^{2}-A_{3} \omega_{0}^{4}\right) \cos \tau_{0} \omega_{0} \\
+\left(\omega_{0}^{5}-A_{2} \omega_{0}^{3}+A_{0} \omega_{0}\right) \sin \tau_{0} \omega_{0}, \\
Q_{I}=\left(A_{1} \omega_{0}^{2}-A_{3} \omega_{0}^{4}\right) \sin \tau_{0} \omega_{0} \\
-\left(\omega_{0}^{5}-A_{2} \omega_{0}^{3}+A_{0} \omega_{0}\right) \cos \tau_{0} \omega_{0} .
\end{gathered}
$$

Obviously, if the condition $\left(H_{3}\right) P_{R} Q_{R}+P_{I} Q_{I} \neq 0$ holds, then $\operatorname{Re}[d \lambda / d \tau]_{\tau=\tau_{0}}^{-1} \neq 0$. Thus, according to the Hopf bifurcation theorem in [21], we have the following results.
Theorem 1. Suppose that the conditions $\left(H_{1}\right)-\left(H_{3}\right)$ hold. The positive equilibrium $D_{*}\left(S_{*}, E_{*}, I_{*}, R_{*}\right)$ of system (3) is asymptotically stable for $\tau \in\left[0, \tau_{0}\right)$. System (3) undergoes a Hopf bifurcation at the positive equilibrium $D_{*}\left(S_{*}, E_{*}, I_{*}, R_{*}\right)$ when $\tau=\tau_{0}$ and a family of periodic solutions bifurcate from the positive equilibrium $D_{*}\left(S_{*}, E_{*}, I_{*}, R_{*}\right)$ near $\tau=\tau_{0}$.

\section{Properties of the Hopf Bifurcation}

In the previous section, we have obtained the conditions for the Hopf bifurcation to occur when $\tau=\tau_{0}$. In this section, we will study properties of the Hopf bifurcation such as direction and stability by using the normal form theory and the center manifold theorem in [21].

Let $u_{1}(t)=S(t)-S_{*}, u_{2}(t)=E(t)-E_{*}, u_{3}(t)=I(t)-I_{*}$, $u_{4}(t)=R(t)-R_{*}$, and $\tau=\tau_{0}+\mu, \mu \in R$ and normalize $t \rightarrow$ $(t / \tau)$. Then system (3) can be transformed into the following form:

$$
\dot{u}(t)=L_{\mu} u_{t}+F\left(\mu, u_{t}\right),
$$

where $u_{t}=\left(u_{1}(t), u_{2}(t), u_{3}(t), u_{4}(t)\right)^{T} \in C=C\left([-1,0], R^{4}\right)$ and $L_{\mu}: C \rightarrow R^{4}$ and $F: R \times C \rightarrow R^{4}$ are given, respectively, by

$$
\begin{gathered}
L_{\mu} \phi=\left(\tau_{0}+\mu\right)\left(\begin{array}{cccc}
a_{11} & a_{12} & a_{13} & 0 \\
a_{21} & a_{22} & a_{23} & 0 \\
0 & a_{32} & a_{33} & 0 \\
0 & 0 & 0 & a_{44}
\end{array}\right) \phi(0) \\
-\left(\tau_{0}+\mu\right)\left(\begin{array}{cccc}
0 & 0 & 0 & b_{14} \\
0 & 0 & 0 & 0 \\
0 & 0 & b_{33} & 0 \\
0 & 0 & b_{43} & b_{44}
\end{array}\right) \phi(-1), \\
F(\mu, \phi)=\left(\tau_{0}+\mu\right)\left(\begin{array}{c}
-\beta \phi_{1}(0) \phi_{3}(0) \\
\beta \phi_{1}(0) \phi_{3}(0) \\
0 \\
0
\end{array}\right) .
\end{gathered}
$$

By the Riesz representation theorem, there exists a $4 \times 4$ matrix function $\eta(\theta, \mu):[-1,0] \rightarrow R^{4}$ whose elements are of bounded variation such that

$$
L_{\mu} \phi=\int_{-1}^{0} d \eta(\theta, \mu) \phi(\theta), \quad \phi \in C .
$$

In fact, we choose

$$
\begin{aligned}
\eta(\theta, \mu)= & \left(\tau_{0}+\mu\right)\left(\begin{array}{cccc}
a_{11} & a_{12} & a_{13} & 0 \\
a_{21} & a_{22} & a_{23} & 0 \\
0 & a_{32} & a_{33} & 0 \\
0 & 0 & 0 & a_{44}
\end{array}\right) \delta(\theta) \\
& -\left(\tau_{0}+\mu\right)\left(\begin{array}{cccc}
0 & 0 & 0 & b_{14} \\
0 & 0 & 0 & 0 \\
0 & 0 & b_{33} & 0 \\
0 & 0 & b_{43} & b_{44}
\end{array}\right) \delta(\theta+1)
\end{aligned}
$$

where $\delta$ is the Dirac delta function. 

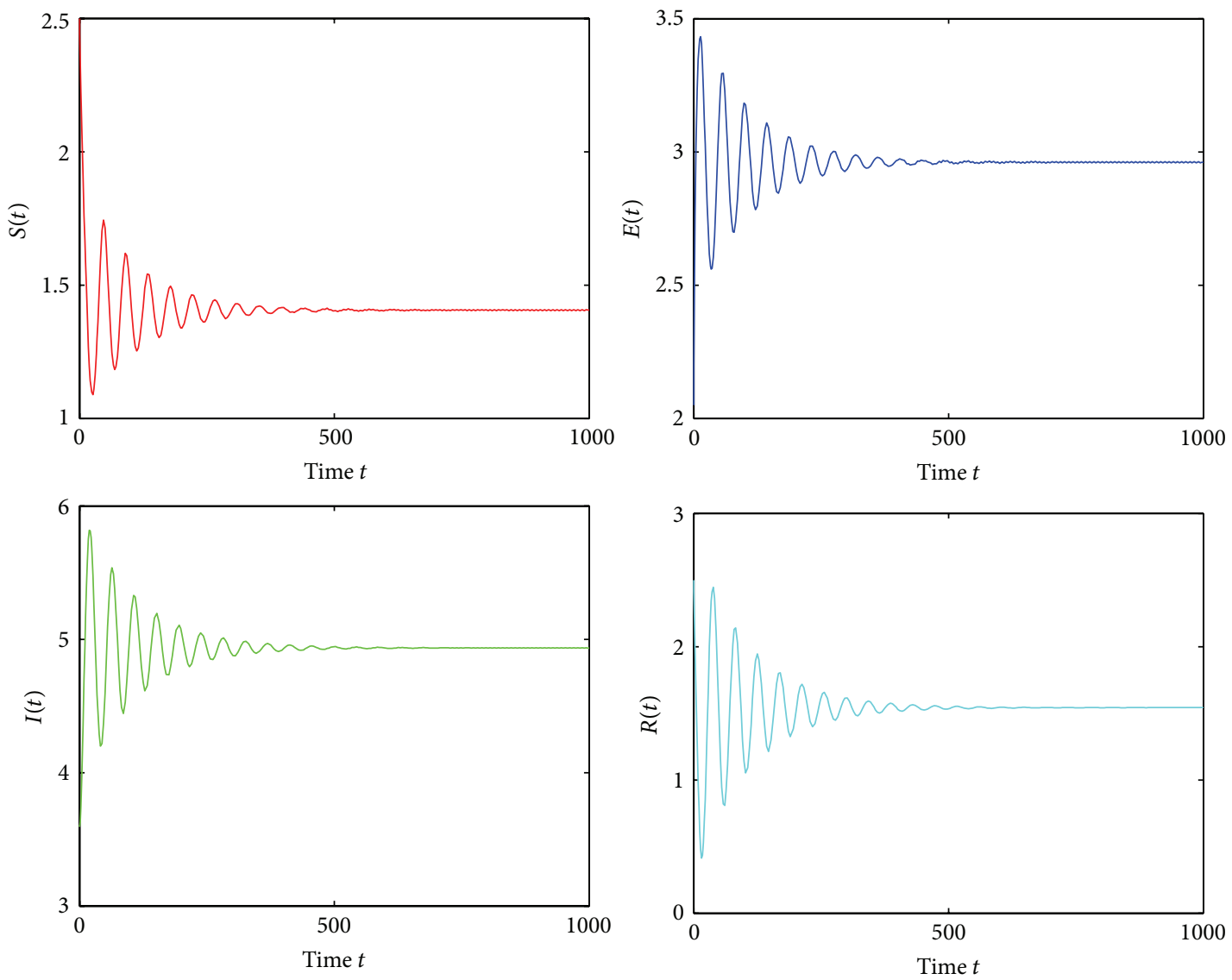

FIGURE 1: The track of the states $S, E, I$, and $R$ for $\tau=12.5000<13.6109=\tau_{0}$.

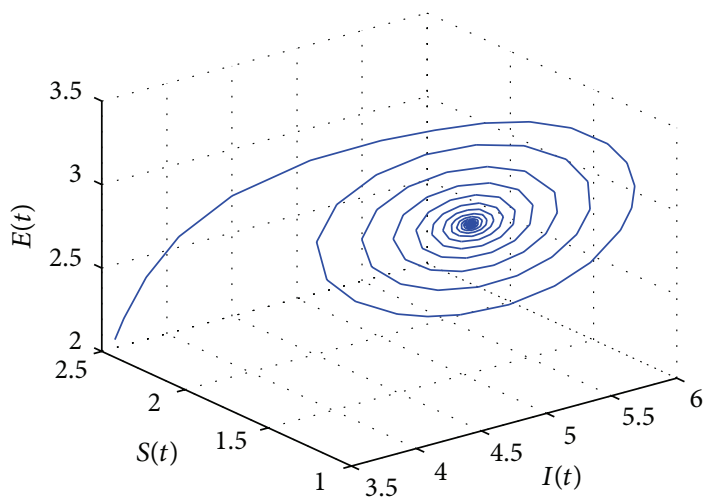

FIGURE 2: The phase plot of the states $S, E$, and $I$ for $\tau=12.5000<$ $13.6109=\tau_{0}$.

For $\phi \in C\left([-1,0], R^{4}\right)$, we define

$$
\begin{gathered}
A(\mu) \phi= \begin{cases}\frac{d \phi(\theta)}{d \theta}, & -1 \leq \theta<0, \\
\text { int }_{-1}^{0} d \eta(\theta, \mu) \phi(\theta), & \theta=0,\end{cases} \\
R(\mu) \phi= \begin{cases}0, & -1 \leq \theta<0, \\
F(\mu, \phi), & \theta=0 .\end{cases}
\end{gathered}
$$

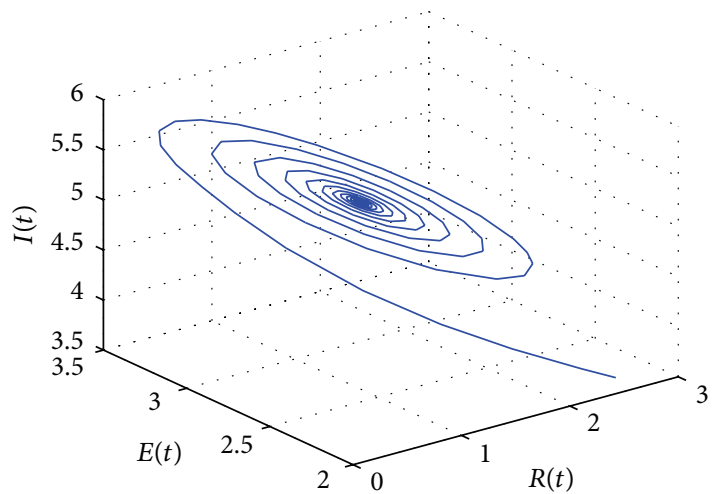

FIgURE 3: The phase plot of the states $E, I$, and $R$ for $\tau=12.5000<$ $13.6109=\tau_{0}$.

Then system (25) can be transformed into the following operator equation:

$$
\dot{u}(t)=A(\mu) u_{t}+R(\mu) u_{t} .
$$

The adjoint operator $A^{*}$ of $A$ is defined by

$$
A^{*}(\varphi)= \begin{cases}-\frac{d \varphi(s)}{d s}, & 0<s \leq 1 \\ \int_{-1}^{0} d \eta^{T}(s, 0) \varphi(-s), & s=0,\end{cases}
$$



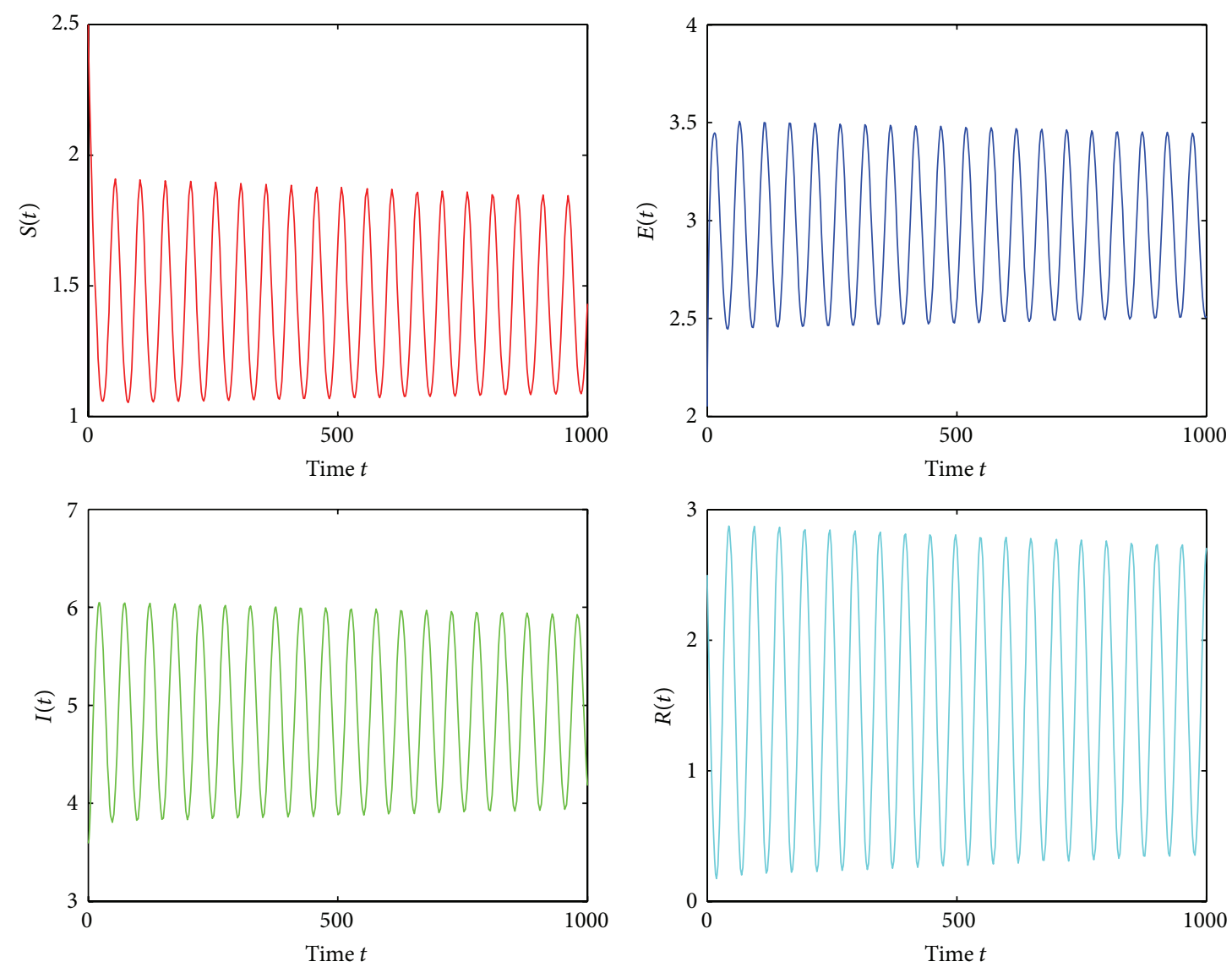

Figure 4: The track of the states $S, E, I$, and $R$ for $\tau=14.9500>13.6109=\tau_{0}$.

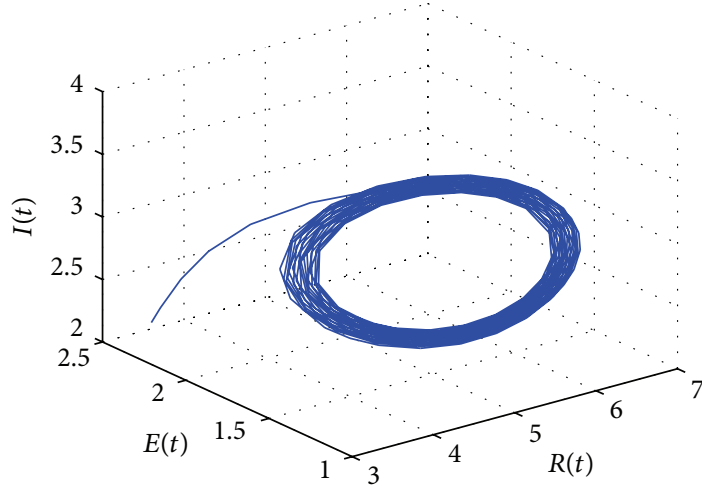

FIgUre 5: The phase plot of the states $S, E$, and $I$ for $\tau=14.9500>$ $13.6109=\tau_{0}$.

associated with a bilinear form:

$$
\begin{aligned}
& \langle\varphi(s), \phi(\theta)\rangle \\
& \quad=\bar{\varphi}(0) \phi(0)-\int_{\theta=-1}^{0} \int_{\xi=0}^{\theta} \bar{\varphi}(\xi-\theta) d \eta(\theta) \phi(\xi) d \xi,
\end{aligned}
$$

where $\eta(\theta)=\eta(\theta, 0)$.

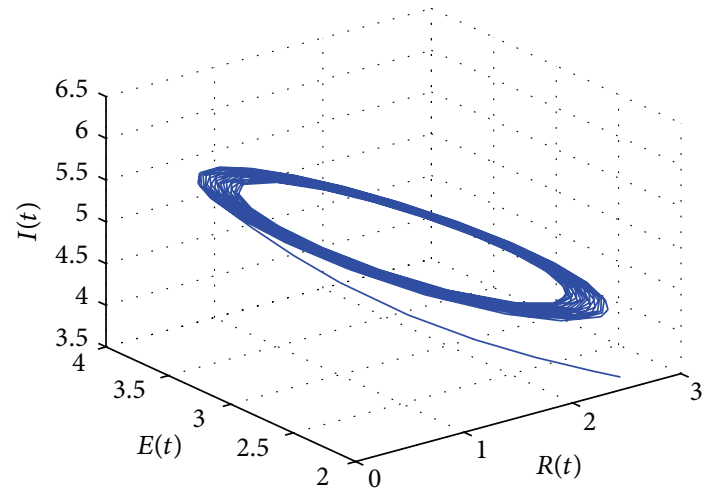

Figure 6: The phase plot of the states $E, I$, and $R$ for $\tau=14.9500>$ $13.6109=\tau_{0}$.

Let $\rho(\theta)=\left(1, \rho_{2}, \rho_{3}, \rho_{4}\right)^{T} e^{i \omega_{0} \tau_{0} \theta}$ be the eigenvector of $A$ corresponding to $i \omega_{0} \tau_{0}$ and let $\rho^{*}(s)=D\left(1, \rho_{2}^{*}, \rho_{3}^{*}, \rho_{4}^{*}\right) e^{i \omega_{0} \tau_{0} s}$ be the eigenvector of $A^{*}$ corresponding to $-i \omega_{0} \tau_{0}$. From the definition of $A(0)$ and $A^{*}(0)$, we can get

$$
\begin{gathered}
\rho_{2}=-\frac{i \omega_{0}-a_{33}-b_{33} e^{-i \tau_{0} \omega_{0}}}{\left(i \omega_{0}-a_{22}\right)\left(i \omega_{0}-a_{33}-b_{33} e^{-i \tau_{0} \omega_{0}}\right)-a_{23} a_{32}}, \\
\rho_{3}=\frac{a_{21} a_{32}}{\left(i \omega_{0}-a_{22}\right)\left(i \omega_{0}-a_{33}-b_{33} e^{-i \tau_{0} \omega_{0}}\right)}
\end{gathered}
$$




$$
\begin{gathered}
\rho_{4}=\frac{b_{43} e^{-\tau_{0} \omega_{0}}}{i \omega_{0}-a_{44}+b_{33} e^{-\tau_{0} \omega_{0}}} \rho_{3}, \\
\rho_{2}^{*}=-\frac{a_{11}}{i \omega_{0}+a_{21}}, \\
r_{3}^{*}=-\frac{\left(i \omega_{0}\left(a_{12}-a_{11}\right)+a_{12} a_{21}-a_{11} a_{22}\right.}{a_{32}\left(i \omega_{0}+a_{21}\right)}, \\
\rho_{4}^{*}=\frac{b_{14} e^{i \tau_{0} \omega_{0}}}{i \omega_{0}+a_{44}+b_{44} e^{i \tau_{0} \omega_{0}}} .
\end{gathered}
$$

From (32), we have

$$
\begin{aligned}
\left\langle\rho^{*}, \rho\right\rangle=\bar{D}[ & 1+\rho_{2} \bar{\rho}_{2}^{*}+\rho_{3} \bar{\rho}_{3}^{*}+\rho_{4} \bar{\rho}_{4}^{*}+\tau_{0} e^{-i \tau_{0} \omega_{0}} \\
& \left.\times\left(\rho_{3}\left(b_{33} \bar{\rho}_{3}^{*}+b_{43} \bar{\rho}_{4}^{*}\right)+\rho_{4}\left(b_{14}+b_{44} \bar{\rho}_{4}^{*}\right)\right)\right] .
\end{aligned}
$$

Then, we choose

$$
\begin{aligned}
\bar{D}= & {\left[1+\rho_{2} \bar{\rho}_{2}^{*}+\rho_{3} \bar{\rho}_{3}^{*}+\rho_{4} \bar{\rho}_{4}^{*}+\tau_{0} e^{-i \tau_{0} \omega_{0}}\right.} \\
& \left.\times\left(\rho_{3}\left(b_{33} \bar{\rho}_{3}^{*}+b_{43} \bar{\rho}_{4}^{*}\right)+\rho_{4}\left(b_{14}+b_{44} \bar{\rho}_{4}^{*}\right)\right)\right]^{-1}
\end{aligned}
$$

such that $\left\langle q^{*}, q\right\rangle=1,\left\langle q^{*}, \bar{q}\right\rangle=0$.
Next, we can obtain the coefficients which will be used to determine the properties of the Hopf bifurcation by following the algorithms introduced in [21] and using a computation process as in [22]:

$$
\begin{gathered}
g_{20}=2 \beta \tau_{0} \bar{D} \rho_{3}\left(\bar{\rho}_{2}^{*}-1\right), \\
g_{02}=2 \beta \tau_{0} \bar{D} \bar{\rho}_{3}\left(\bar{\rho}_{2}^{*}-1\right), \\
\left.g_{11}=\beta \tau_{0} \bar{D}\left(r_{3}+\bar{r}_{3}\right)\left(\bar{r}_{2}^{*}-1\right)\right), \\
g_{21}=2 \beta \tau_{0} \bar{D}\left(\bar{\rho}_{2}^{*}-1\right)\left(W_{11}^{(1)}(0) \rho_{3}+\frac{1}{2} W_{20}^{(1)}(0) \bar{\rho}_{3}\right. \\
\left.+W_{11}^{(3)}(0)+\frac{1}{2} W_{20}^{(3)}(0)\right),
\end{gathered}
$$

with

$$
\begin{gathered}
W_{20}(\theta)=\frac{i g_{20} q(0)}{\tau_{0} \omega_{0}} e^{i \tau_{0} \omega_{0} \theta}+\frac{i \bar{g}_{02} \bar{q}(0)}{3 \tau_{0} \omega_{0}} e^{-i \tau_{0} \omega_{0} \theta}+E_{1} e^{2 i \tau_{0} \omega_{0} \theta} \\
W_{11}(\theta)=-\frac{i g_{11} q(0)}{\tau_{0} \omega_{0}} e^{i \tau_{0} \omega_{0} \theta}+\frac{i \bar{g}_{11} \bar{q}(0)}{\tau_{0} \omega_{0}} e^{-i \tau_{0} \omega_{0} \theta}+E_{2}
\end{gathered}
$$

where $E_{1}$ and $E_{2}$ can be determined by the following equations, respectively:

$$
\begin{aligned}
& E_{1}=2\left(\begin{array}{cccc}
2 i \omega_{0}-a_{11} & -a_{12} & -a_{13} & -b_{14} e^{-2 i \omega_{0} \tau_{0}} \\
-a_{21} & 2 i \omega_{0}-a_{22} & -a_{23} & 0 \\
0 & -a_{32} & 2 i \omega_{0}-a_{33}-b_{33} e^{-2 i \omega_{0} \tau_{0}} & 0 \\
0 & 0 & -b_{43} e^{-2 i \omega_{0} \tau_{0}} & 2 i \omega_{0}-a_{44}-b_{44} e^{-2 i \omega_{0} \tau_{0}}
\end{array}\right)\left(\begin{array}{c}
-1 \\
E_{1}^{(1)} \\
E_{1}^{(2)} \\
0 \\
0
\end{array}\right) \text {, } \\
& E_{2}=-\left(\begin{array}{cccc}
a_{11} & a_{12} & a_{13} & b_{14} \\
a_{21} & a_{22} & a_{23} & 0 \\
0 & a_{32} & a_{33}+b_{33} & 0 \\
0 & 0 & b_{43} & a_{44}+b_{44}
\end{array}\right)^{-1}\left(\begin{array}{c}
E_{2}^{(1)} \\
E_{2}^{(2)} \\
0 \\
0
\end{array}\right),
\end{aligned}
$$

with

$$
\begin{array}{cl}
E_{1}^{(1)}=-\beta \rho_{3}, & E_{1}^{(2)}=\beta \rho_{3}, \\
E_{2}^{(1)}=-\beta\left(\rho_{3}+\bar{\rho}_{3}\right), & E_{2}^{(2)}=\beta\left(\rho_{3}+\bar{\rho}_{3}\right) .
\end{array}
$$

Therefore, we can calculate the following values:

$$
\begin{gathered}
C_{1}(0)=\frac{i}{2 \tau_{0} \omega_{0}}\left(g_{11} g_{20}-2\left|g_{11}\right|^{2}-\frac{\left|g_{02}\right|^{2}}{3}\right)+\frac{g_{21}}{2}, \\
\mu_{2}=-\frac{\operatorname{Re}\left\{C_{1}(0)\right\}}{\operatorname{Re}\left\{\lambda^{\prime}\left(\tau_{0}\right)\right\}}
\end{gathered}
$$

$$
\begin{gathered}
\beta_{2}=2 \operatorname{Re}\left\{C_{1}(0)\right\}, \\
T_{2}=-\frac{\operatorname{Im}\left\{C_{1}(0)\right\}+\mu_{2} \operatorname{Im}\left\{\lambda^{\prime}\left(\tau_{0}\right)\right\}}{\tau_{0} \omega_{0}} .
\end{gathered}
$$

Based on the discussion above, we can obtain the following results.

Theorem 2. For system (3), if $\mu_{2}>0\left(\mu_{2}<0\right)$, the Hopf bifurcation is supercritical (subcritical). If $\beta_{2}<0\left(\beta_{2}>0\right)$, the bifurcating periodic solutions are stable (unstable). If $\mathrm{T}_{2}>$ $0\left(T_{2}<0\right)$ the period of the bifurcating periodic solutions increases (decreases). 


\section{Numerical Simulation}

In this section, we present a numerical example to verify the theoretical analysis in Sections 2 and 3. Let $b=0.7, d=0.06$, $p=0.02, q=0.01, \beta=0.1, \varepsilon=0.2, \gamma=0.05, \eta=0.01$, and $\zeta=0.1$. We can get a particular case of system (3):

$$
\begin{aligned}
& \frac{d S(t)}{d t}=0.7-0.1 I(t) S(t)-0.014 E(t)-0.007 I(t) \\
& -0.06 S(t)+0.1 R(t-\tau), \\
& \frac{d E(t)}{d t}=0.1 I(t) S(t)+0.014 E(t)+0.007 I(t) \\
& -0.2 E(t)-0.06 E(t), \\
& \frac{d I(t)}{d t}=0.2 E(t)-0.05 I(t-\tau)-0.06 I(t) \\
& -0.01 I(t) \\
& \frac{d R(t)}{d t}=0.05 I(t-\tau)-0.06 R(t)-0.1 R(t-\tau) .
\end{aligned}
$$

Then, we can get that $R_{0}=7.5774>1,(\varepsilon+d)(d+\gamma+\eta)=$ $0.0312>p b(d+\gamma+\eta)+q b \varepsilon=0.0031$, and system (41) has a unique positive equilibrium $D_{*}(1.4050,1.5422,4.9350$, and 2.9610). Further, we have $D_{1}=1.0795>0, D_{2}=$ $0.3360>0, D_{3}=0.0141>0$, and $D_{4}=2.8200 e-$ $004>0$. That is, condition $\left(H_{1}\right)$ holds. Thus, we can obtain $\omega_{0}=0.1245, \tau_{0}=13.6109$, and $\lambda^{\prime}\left(\tau_{0}\right)=0.0073+0.0299 i$. Therefore, from Theorem 1 , the positive equilibrium $D_{*}$ is asymptotically stable when $\tau \in[0,13.6109)$ and unstable when $\tau>13.6109$. As can be seen from Figures 1, 2, and 3 , when $\tau=12.5 \in[0,13.6109)$, the positive equilibrium $D_{*}$ is asymptotically stable. However, when $\tau=14.95>$ 13.6109 , the positive equilibrium $D_{*}$ will lose its stability, a Hopf bifurcation occurs, and a family of periodic solutions bifurcate from the positive equilibrium $D_{*}$. This property can be illustrated by Figures 4, 5, and 6. In addition, from (40), we can get $C_{1}(0)=-0.00904+0.6833 i, \mu_{2}=12.3836>$ $0, \beta_{2}=-0.1808<0$, and $T_{2}=-0.6217<0$. Thus, according to Theorem 2, we know that the Hopf bifurcation is supercritical. The bifurcating periodic solutions are stable and the period of the periodic solutions decreases.

\section{Conclusions}

In this paper, we incorporate the time delay due to the period the antivirus software has to use to clean the worms in one node and the temporary immunity period of the recovered nodes into the model considered in the literature [6] and get a delayed SEIRS epidemic model for the transmission of worms in computer network through vertical transmission. The effects of the time delay on the dynamics of the model are investigated. It is found that the time delay can play a complicated role on the model by analyzing the distribution of the roots of the associated characteristic equation. When the time delay is suitable small, the positive equilibrium is asymptotically stable. However, a local Hopf bifurcation occurs and a branch of periodic solutions bifurcates from the positive equilibrium when the delay passes through the critical value $\tau_{0}$. Furthermore, the properties of the Hopf bifurcation such as direction and stability are determined by using the normal form theory and center manifold theorem. In order to verify the theoretical analysis, a numerical example is also included.

\section{Acknowledgments}

This work was supported by the National Natural Science Foundation of China (61273070), a project funded by the Priority Academic Program Development of Jiangsu Higher Education Institution, Major Science Foundation Subject for the Education Department of Anhui Province under Project no. ZD200905, and a Project funded by the Ministry of Education (12YJA630136).

\section{References}

[1] J. O. Kephart and S. R. White, "Measuring and modeling computer virus prevalence," in Proceedings of the IEEE Computer Society Symposium on Research in Security and Privacy, pp. 2-15, May 1993.

[2] J. O. Kephart and S. R. White, "Directed-graph epidemiological models of computer viruses," in Proceedings of the IEEE Computer Society Symposium on Research in Security and Privacy, pp. 343-358, May 1991.

[3] R. W. Thommes and M. J. Coates, "Modeling virus propagation in peer-to-peer networks," in Proceedings of the IEEE International Conference on Information, Communications and Signal Processing, pp. 981-985, December 2005.

[4] C. C. Zou, D. Towsley, and W. Gong, "On the performance of Internet worm scanning strategies," Performance Evaluation, vol. 63, no. 7, pp. 700-723, 2006.

[5] H. Yuan and G. Chen, "Network virus-epidemic model with the point-to-group information propagation," Applied Mathematics and Computation, vol. 206, no. 1, pp. 357-367, 2008.

[6] B. K. Mishra and S. K. Pandey, "Dynamic model of worms with vertical transmission in computer network," Applied Mathematics and Computation, vol. 217, no. 21, pp. 8438-8446, 2011.

[7] J. C. Wierman and D. J. Marchette, "Modeling computer virus prevalence with a susceptible-infected-susceptible model with reintroduction," Computational Statistics and Data Analysis, vol. 45, no. 1, pp. 3-23, 2004.

[8] Y. B. Kafai and S. White, "Understanding virtual epidemics: children's folk conceptions of a computer virus," Journal of Science Education and Technology, vol. 17, no. 6, pp. 523-529, 2008.

[9] F. W. Wang, Y. K. Zhang, C. G. Wang, J. Ma, and S. Moon, "Stability analysis of a SEIQV epidemic model for rapid spreading worms," Computers and Security, vol. 29, no. 4, pp. 410-418, 2010.

[10] J. R. C. Piqueira and V. O. Araujo, "A modified epidemiological model for computer viruses," Applied Mathematics and Computation, vol. 213, no. 2, pp. 355-360, 2009.

[11] Y. Yao, X. W. Xie, H. Guo, G. Yu, F. X. Gao, and X. J. Tong, "Hopf bifurcation in an Internet worm propagation model with time delay in quarantine," Mathematical and Computer Modelling, vol. 57, pp. 2635-2646, 2013. 
[12] J. G. Ren, X. F. Yang, L. X. Yang, Y. Xu, and F. Yang, "A delayed computer virus propagation model and its dynamics," Chaos, Solitons and Fractals, vol. 45, no. 1, pp. 74-79, 2012.

[13] H. Y. Bai and Y. H. Zhai, "Hopf bifurcation analysis for the model of the chemostat with one species of organism," Abstract and Applied Analysis, vol. 2013, Article ID 829045, 7 pages, 2012.

[14] L. Feng, X. Liao, H. Li, and Q. Han, "Hopf bifurcation analysis of a delayed viral infection model in computer networks," Mathematical and Computer Modelling, vol. 56, no. 7-8, pp. 167$179,2012$.

[15] G. Zhu and J. Wei, "Stability and Hopf bifurcation analysis of coupled optoelectronic feedback loops," Abstract and Applied Analysis, vol. 2013, Article ID 918943, 11 pages, 2013.

[16] B. Wang, P. Shi, H. R. Karimi, and C. C. Lim, "Observerbased sliding mode control for stabilization of a dynamic system with delayed output feedback," Mathematical Problems in Engineering, vol. 2013, Article ID 537414, 6 pages, 2013.

[17] T. L. Zhang, J. L. Liu, and Z. D. Teng, "Stability of Hopf bifurcation of a delayed SIRS epidemic model with stage structure," Nonlinear Analysis. Real World Applications, vol. 11, no. 1, pp. 293-306, 2010.

[18] R. Wang and J. Li, "Adaptive neural control for a class of outputs time-delay nonlinear systems," Mathematical Problems in Engineering, vol. 2012, Article ID 852161, 16 pages, 2012.

[19] G. Zhu and J. J. Wei, "Synchronized Hopf bifurcation analysis in a delay-coupled semiconductor lasers system," Journal of Applied Mathematics, vol. 2012, Article ID 257635, 20 pages, 2012.

[20] W. L. Zhu, X. F. Ruan, Y. Qin, and J. Zhuang, "Exponential stability of stochastic nonlinear dynamical price system with delay," Mathematical Problems in Engineering, vol. 2013, Article ID 168169, 9 pages, 2013.

[21] B. D. Hassard, N. D. Kazarinoff, and Y. H. Wan, Theory and Applications of Hopf Bifurcation, Cambridge University Press, Cambridge, UK, 1981.

[22] C. J. Xu and X. F. He, "Stability and bifurcation analysis in a class of two-neuron networks with resonant bilinear terms," Abstract and Applied Analysis, vol. 2011, Article ID 697630, 21 pages, 2011. 


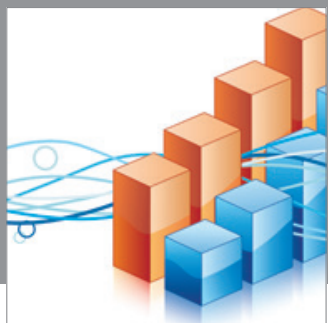

Advances in

Operations Research

mansans

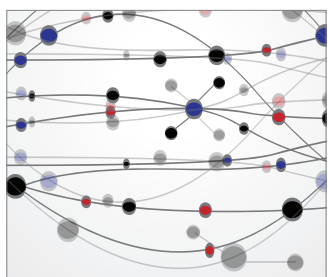

The Scientific World Journal
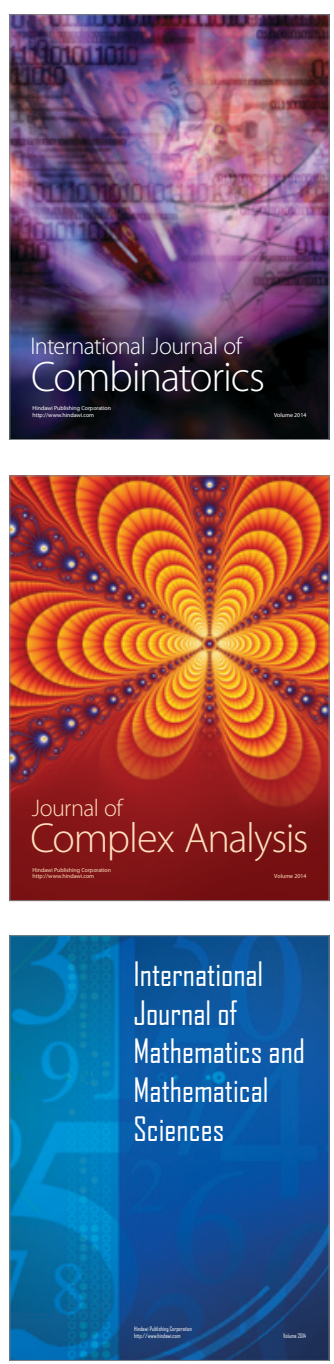
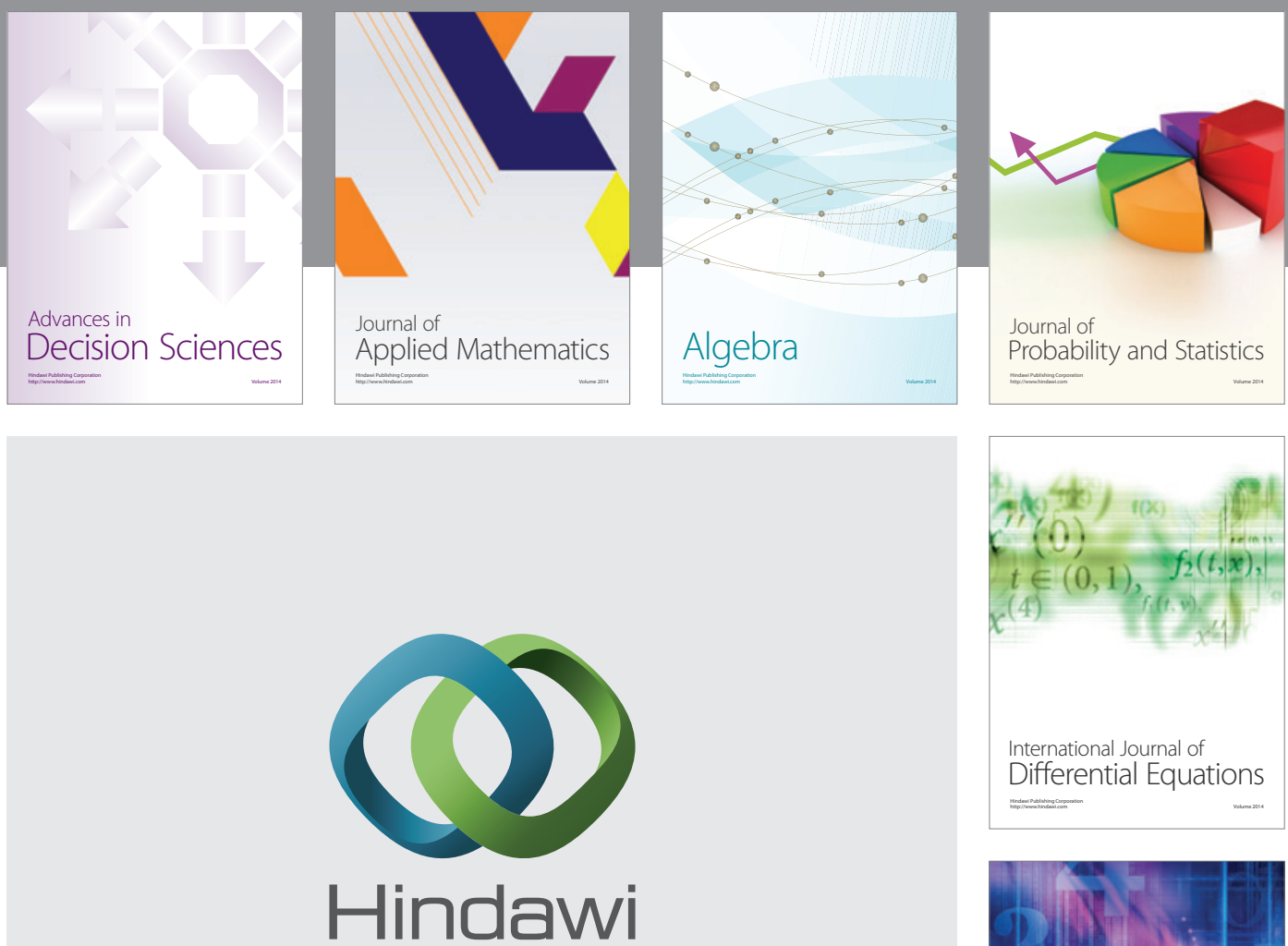

Submit your manuscripts at http://www.hindawi.com
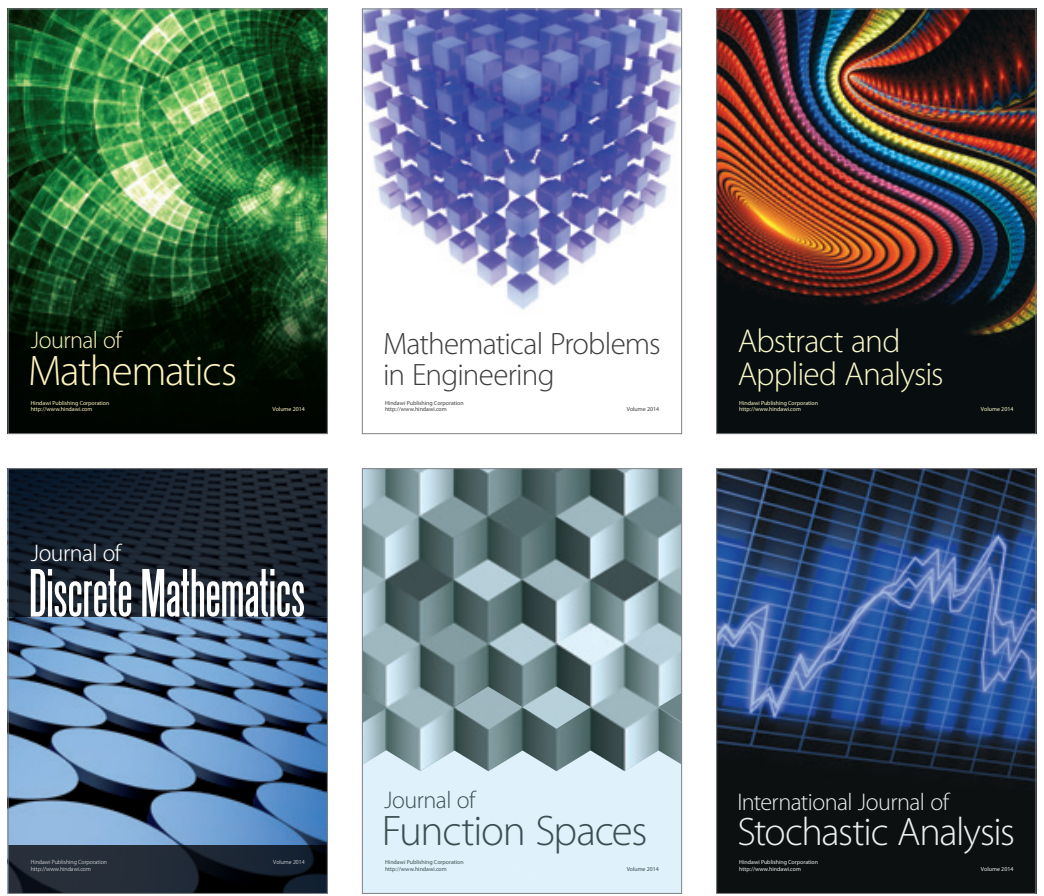

Journal of

Function Spaces

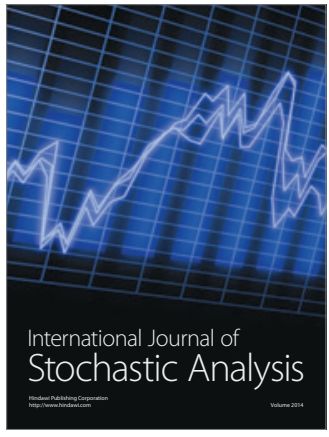

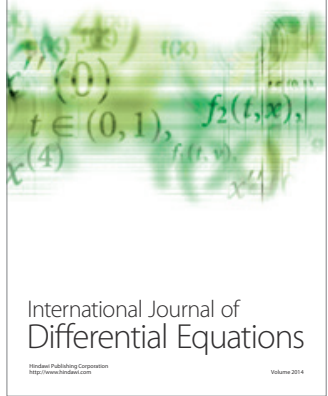
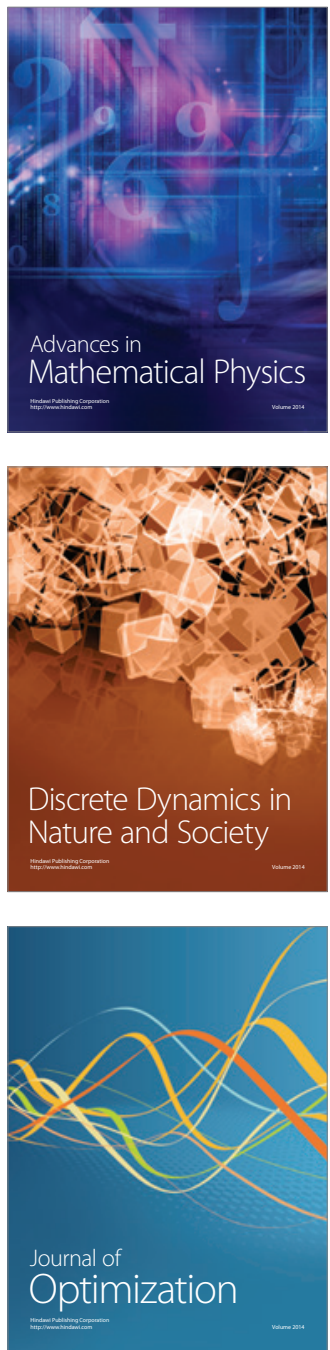